\title{
Biomedical Device for Spasticity Quantification Based on the Velocity Dependence of the Stretch Reflex Threshold
}

\author{
João Ferreira \\ University of Minho \\ Braga, Portugal \\ egjferreira@gmail.com \\ Vitor Moreira \\ Hospital of Braga \\ Braga, Portugal \\ vitorvarandasmoreira@gmail.com
}

\begin{abstract}
Spasticity is a common and complex motor disorder that affects more than 12 million persons in the world. There are several studies on spasticity quantification in the literature but there is still a need for measurement improvements. This paper presents the validation, in clinical environment, of a mechatronic medical device, dedicated, and specially designed and constructed for spasticity quantification, in joint of ankle, elbow and knees. This approach is based on the velocity-dependent of the Stretch Reflex threshold. The relevant variables, the measurement range and the adequate measurement systems are indicated. The reliability of the developed mechatronic medical system is confirmed by data acquisition and analysis, following a systematic methodology, also presented in the paper.
\end{abstract}

\section{Introduction}

Spasticity is a complex motor disorder due to a supraspinal inhibition, resulting from a hyper-excitability of the stretch reflex (SR) [1]. Spasticity affects more than 12 million persons in the world [2], and is always seen in patient with upper motor neuron (UMN) dysfunctions such as cerebral vascular accidents, spinal cord injuries, and multiple sclerosis. The mechanism of spasticity is commonly thought as an exaggerated SR, which is a velocity-dependent increase in the resistance to the passive movement [3]. The SR Threshold is significantly reduced in spastic muscles, and this reduction is correlated to the increase in reflex joint torque [4]. Spasticity in conjugation with excessive muscle tone frequently interferes in the voluntary motor function, causing difficulties in daily activities [3]. Some of the common symptoms are: a change in the recruitment of limb's segments and a severe mal-functioning of the tendons reflex.

\author{
José Machado \\ University of Minho \\ Guimarães, Portugal \\ jmachado@dem.uminho.pt \\ Filomena Soares \\ University of Minho \\ Braga, Portugal \\ fsoares@dei.uminho.pt
}

Spasticity is a serious neurological disorder and several treatments and therapies have emerged for its treatment [5]. The treatment must be appropriate to the symptoms presented by each patient and the success of treatment is strongly dependent of the selection of patients [6]. Therefore, the existence of a sensitive, consistent and reproducible method for clinical evaluation, of the level of spasticity, plays an important role in the selection of patients to access appropriate therapies [5], [7].

Spasticity quantification is a hard and complex task [8]. The correct quantification of spasticity has been under an extensive study by the scientific community, but there is not yet available a well-accepted standard method for spasticity determination and quantification. The literature presents several methods for spasticity quantification: The Ashworth Scale (AS) and the Ashworth modified version (MAS); Isokinetic device with generator torque; Pendulum Test. None of them is fully accepted, due to various reasons [2], [7], [8]. The AS and the MAS are the common scales in clinical quantifications of spasticity, despite experts agree that both scales may not measure the characteristics that distinguish spasticity from other tonus disorders. Although the scale is useful in determining the amount of resistance felt in the passive displacement of the limbs, it does not quantify the dependence to velocity of $\mathrm{SR}$, which is the feature that differentiates spasticity [7]. This scale has a low reproductive rate, a lack of validation in all muscle groups, usually affected by spasticity [1].

The approach of traditional measures is based on the phase and magnitude of the tonic SR and the resistance to passive stretch. Nevertheless, this measure is not correlated to the clinical impression of the spasticity level, inability to differentiate the mechanical stiffness from the reflective stiffness and the implementation of the device is still complex; also, the measurement does not meet the criteria of the known theory. Hence, there is 
still a need for a device that meets these requirements [1], [7], [8].

The key issue is to determine which variables are necessary to correctly quantify this disorder. A correct measure to quantify spasticity must follow the physiological mechanisms related to the stand-up position control and the movement in healthy individuals and/or must detect possible deficiencies in any of these mechanisms that lead to motor disorders. For the acceptance of the method, their approach must be in accordance to a standard spasticity definition [7], [8]. The work presented in [9] defined spasticity as: "a motor disorder characterized by a velocity-dependent increase in tonic stretch reflexes ("muscle tonus") with exaggerated tendon jerk, resulting in hyper excitability of the stretch reflexes, as one of the component of the upper motor neuron syndrome". This definition is still accepted nowadays; it includes some important aspects: it refers to spasticity as a symptom, as a disorder in the somatic mobility, related to the high tonic component of the SR; it is due to the spinal reflex; it is one of the symptoms of the upper motoneuron syndrome; the tonic stretch is associated to the exaggerated tendon jerks, and reflects the physical component of the SR; the reflex of the tone stretch is the basis of the tonus; it is referred that the excess of the reflex depends on the stretch velocity [10]. This last statement can be the key issue for spasticity quantification [2].

This paper presents the development of a dedicated mechatronic medical device for quantification of all levels of spasticity, in joint of ankle, elbow or knees. This approach is based on the velocity-dependent of the SR threshold.

\section{Proposed approach}

\subsection{Device}

The SR is an involuntary contraction elicited by a brief stimulus to muscle receptors. If the arm and muscle are immobilized the result will be a measurable change in the tension of the tendon [4].

On this approach the method is focused on the velocity-dependent increase in the SR Threshold, according to the criteria of standard definition of spasticity, proposed by Lance [9]. This definition suggested that the Stretch Reflex Threshold (SRT) depends on the velocity of stretch.

It is determined the angle of biomechanics range and the angular velocity when an increase in electromyography activity occurs; it is further processed by a custom developed software. Most of the daily life activity requires joint angles of $45^{\circ}$ for ankle, $140^{\circ}$ to full flexion to the knee [11] and $150^{\circ}$ for elbow [12]. These ranges should be considered to ensure the assessment of movement in the whole biomechanical range, recruited in daily life. The study proposed in [2], where patients with stroke are studied, there is detected an increase of activity of the EMG (electromyography) signal in Biceps Brachia, due to stretching the elbow joint, at angular velocities of $51 \% \mathrm{~s}, 161 \%$ and $430 \%$ s; this demonstrates the velocity-dependence of SRT, in muscles affected by spasticity.

To determine the SR Threshold we propose three measurements: EMG signal activity in the muscle; angular velocity of passive muscle stretch and the joint angle position. The angular position and angular velocity are measured using an electro-goniometer. The EMG signal is recorded using surface $\mathrm{Ag} / \mathrm{AgCL}$ electrodes with bipolar configuration. The EMG and the goniometer signals were filtered and sampled with an analogical-to-digital converter (ADC) and sent to the computer for storage and processing data.

\subsection{Patients}

The patients' selection was based on the occurrence of spasticity symptoms in the flexor muscles in the elbow. It was assured, by the clinical assistant that patients selected were not under the influence of any medicine; this fact could interfere in the results due to possible influence in neuromuscular functions. The selected patients were aged between 37 and 86 years old.

All the patients received the necessary information regarding the experimental procedure and research objective. After reflecting on their participation in the study, they signed the Informed Consent.

\subsection{Experimental methodology}

Each patient was evaluated in three consecutive sessions, separated by two to five days. In each evaluation, it was defined the initial position of the elbow joint, the position corresponding to the maximum allowable bending of the arm and forearm, with no contact between segments. The goniometer was attached to the patient member to be assessed. A goniometer arm was aligned with the patient's forearm while the other goniometer arm was aligned with the patient's arm. Then, the surface EMG electrodes were placed in correspondence to the motor point of the muscle.

After checking the initial conditions, it was started the process of recording the EMG signals and angular displacements, by manually starting stretching the muscles. The forearm was extended, by the evaluator, at different velocities, from its initial position to the maximum extent allowed (specific to each patient). The tests promoted ten stretches at "low velocity" ( $2 \%$ second to $150 \%$ second), ten stretches at "moderate velocity" $\left(151^{\circ}\right.$ second to $300 \%$ second) and ten stretches at "high velocity (higher than $300 \%$ second).

\section{Results}

The time instant at which a SR occurred corresponds to the time instant where a sustained increase of the EMG activity is verified, two standard deviations (SDs) 
above the signal average that is observed without movement of the patient's arm. This systematic analysis allowed determining, accurately, the corresponding values of angle and angular velocity. The DSRT was defined considering both: the angle of the joint and the value of the angular velocity. For each evaluation, DSRT were presented in a graph in two dimensions, considering: angular velocity (vertical axis) versus joint angle (horizontal axis). Tonic stretch Reflex Threshold (TSRT) was estimated by calculating a linear regression through the DSRT. The value of TSRT was obtained at the point where the regression line intersects the axis angle (horizontal axis). Concerning each regression line, it was considered the coefficient of determination $\left(\mathrm{R}^{2}\right)$, the inclination of the line, the number of DSRTs and it was considered, too, a confidence interval of 95\%. In this analysis, it was considered significant determination coefficient with values equal to or greater than 0.2 and negligible determination coefficients with values below 0.1 [1]. The coefficients between 0.1 and 0.2 are considered as minor.

As expected, SR responds linearly to muscle stretch proportionally to stretch velocity. Figure 1 illustrates an example of TSRT estimation, for patient M19 (Table 1). It can be observed the distribution of 28 DSRTs presented as small circles that allowed the calculation of the regression line, following the methodology presented before.

It can be stated that the regression line intersects the axis corresponding to angular displacement of $124^{\circ}$, clearly inside the range of joint movements. In this case, the regression line presents a slope of $-13,6$ and a determination coefficient of 0,91. DSRT can be observed in all velocities ranges; in particular, at high velocities DSRT occurs at lower muscular length. This fact presents the obtained results, for all the patients, considering the three evaluation sessions.

The results show satisfactory coefficients of determination and a satisfactory variability between evaluation sessions. The patients present significant determination coefficients comprised between $\mathrm{R}^{2}=0.33$ and $\mathrm{R}^{2}=0.9$, with exception of patient M15, that only present significant determination coefficients during the first and second evaluation sessions. The patients M9, M3, M18, M4, M6, M14 and M19 show a low variability between the evaluation sessions, in the TSRT, and standard deviations between \pm 3.5 and \pm 13.5 . The patients M5, M15, M24 e M8 show a high variability between the evaluation sessions and standard deviations between \pm 24.09 e \pm 31.78 .

Considering this last group, the patient M5 has a significant determination coefficient during the three sessions but shows a high variability; it can be explained by the wide discrepancy in the number of observed DSRTs, suggesting a lower excitability of the Upper Motor Neuron (UMN) in the third evaluation session, justifying a higher value of TSRT. Considering patient
M15, there is a negligible factor in determining the third assessment session, $\mathrm{R}^{2}=0.11$, originated by the presence of false DSRTs, leading to a change in the estimated TSRT. Changes in excitability of UMN may be associated to emotional stress, discomfort and high levels of anxiety related to his participation in the experimental tasks [1][2][3]. Low $\mathrm{R}^{2}$ values may be associated to the determination of false DSRTs, due to the presence of artefacts [1]; and errors of handling the goniometer which can cause errors up to $8^{\circ}$ [3].

Considering, for instance, the measured data, corresponding to patient M19 (which results are presented in Figure 2), it can be stated that TSRT (TSRT $=124^{\circ}$ ) is placed inside the range of normal movements of the joint, suggesting that this is the point at which a minimum velocity of stretching, close to zero, would originate a response of SR. According to the model of motor control, "Equilibrium-point", the value of TSRT represents the muscle length from which the muscle is fully activated, in response to any stretch, registering an increase of torque due to the increase of muscle length and of velocity of stretching [12]. The area on the right side of the regression line, which originates a TSRT, corresponds to the area in which any muscular stretch would originate a spastic reaction to the movement. In this case, even with slower velocities of stretching, the range of movement is limited to $124^{\circ}$ of amplitude, showing a wide range of movements compromised by spasticity. This approach allows clinicians to have a clear perception of the range of movements compromised by spasticity. Under this classification a low value of TSRT, near the beginning of the joint range of motion, indicates a high level of spasticity, showing a large range of movements compromised by spasticity [12]; a high value of TSRT, near or above the end of the range of joint movement, indicates a low degree of spasticity, showing a small range of movement compromised by spasticity. Moreover, this study presents the results obtained using a medical device, not very complex, easy to use and to handle.

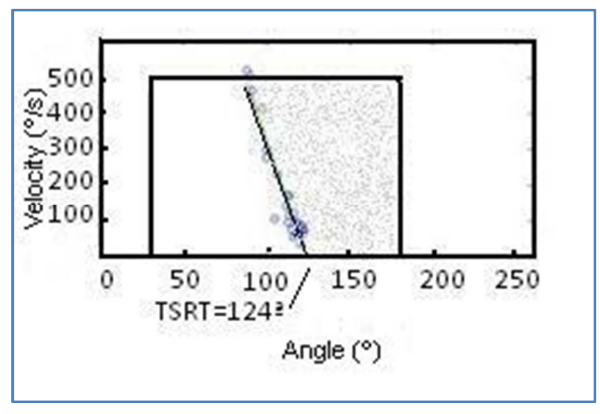

figure 1 Example of TSRT estimation 
Table 1Obtained results

\begin{tabular}{|c|c|c|c|c|c|c|c|c|c|c|c|c|}
\hline \multirow[t]{2}{*}{ Subjects } & \multicolumn{4}{|c|}{ First test } & \multicolumn{4}{|c|}{ Second test } & \multicolumn{4}{|c|}{ Third test } \\
\hline & DSRT & TSRT & Slope & $\mathrm{R}^{2}$ & DSRT & TSRT & Slope & $\mathrm{R}^{2}$ & DSRT & TSRT & Slope & $\mathrm{R}^{2}$ \\
\hline M9 & 13 & 162 & -3.69 & 0.7 & 7 & 169 & -10.74 & 0.71 & 22 & 168 & -3.66 & 0.35 \\
\hline M3 & 14 & 155 & -3.61 & 0.47 & 15 & 178 & -3.92 & 0.33 & 14 & 167 & -6.75 & 0.46 \\
\hline M5 & 21 & 142 & -8.08 & 0.63 & 29 & 155 & -8.16 & 0.58 & 7 & 199 & -2.5 & 0.73 \\
\hline M15 & 15 & 152 & -5.61 & 0.68 & 30 & 161 & -6.37 & 0.54 & 16 & 211 & -2.61 & 0.11 \\
\hline M18 & 23 & 183 & -3.21 & 0.65 & 29 & 182 & -5.78 & 0.77 & 33 & 192 & -4.44 & 0.54 \\
\hline M24 & 9 & 182 & -8.59 & 0.5 & 7 & 222 & -3.76 & 0.82 & 7 & 173 & -3.81 & 0.42 \\
\hline M4 & 15 & 178 & -1.2 & 0.79 & 7 & 168 & -2.84 & 0.69 & 19 & 173 & -7.68 & 0.59 \\
\hline M6 & 27 & 147 & -3.98 & 0.73 & 6 & 154 & -1.57 & 0.56 & 14 & 171 & -4.13 & 0.5 \\
\hline M8 & 20 & 164 & -0.081 & 0.8 & 22 & 169 & -0.006 & 0.75 & 10 & 208 & -1.38 & 0.57 \\
\hline M14 & 14 & 192 & -2.1 & 0.54 & 12 & 195 & -1.6 & 0.19 & 8 & 188 & -7.41 & 0.47 \\
\hline M19 & 7 & 136 & -11.02 & 0.64 & 41 & 151 & -3.91 & 0.52 & 28 & 124 & $-13,6$ & 0.91 \\
\hline
\end{tabular}

testing of spasticity, in Annales de la Réadaptation et de

\section{Conclusions and future work}

The objective of the work was to demonstrate and validate a dedicated biomedical device developed, specially, for quantification of different levels of spasticity, based on the velocity-dependent of the SR threshold.

Spasticity quantification has, as main gap, the dependence to different parameters related to each human being (physiological characteristics), and, also, the insufficient reliability of the systems used in its determination. This study allows to confirm the reliability of the developed system, used for spasticity quantification, and to reduce, considerably, possible errors, mistakes and/or deviations in its quantification. These results are, mainly, consequence of two factors: first, the recent technology used concerning the system development and, second, the development of a dedicated algorithm for muscle activation detection (not specified, in detail, in this paper).

The obtained results show that the proposed biomedical system is robust and reliable concerning spasticity variability. Nevertheless, we consider relevant, as future work, to analyze a larger universe of patients to verify the repeatability of the equipment.

\section{References}

[1] . Le Carvozin, X. Hemot, O. Bartier, G. Carrault, F. Chagneau, P. Callien, H. Allain, P. Rochcongar, Evaluation of pendulum
Médecine Physiqu, Mars. 2002, 45, pp. 510-516.

[2] A. Calota, A.G. Feldman, M.F. Levin, Spasticity measurement based on tonic stretch reflex threshold in stroke using a portable device. In Clinical Neurophysiology. 2008, 119, pp. 2329-2337.

[3] J.J Chen, Y.N. Wu, S.C Huang, H.M. Lee and Y.L. Wangs, The Use of a Portable Muscle Tone Measurement Device to Measure the Effects of Botulinum Toxin Type A on Elbow Flexor Spasticity. Arch Phys Med Rehabil,. Vol. 86, August. 2005.

[4] M. Chardon, Nina L, Surech and w.z. Ryner. A New Method For Reflex Threshold Estimation in Spastic Muscles. Presented at the conference of the IEEE EMBS. Sep. 2009

[5] K. Lee, L. Carson, E. Kinnin, V. Patterson..The Ashworth Scale: A Reliable and Reporducible Method of Measuring Spasticity. Neurorehabilitation \& Neural Repair. 1989, Pp 2005-2009.

[6] R. Oderson,, G. H. Kroft,.APPLICATIONS OF BOTULUNUM TOXIN IN PHYSICAL MEDICINE AND REHABILITATION, Physical Medicine And Rehabilitation Clinics of North America, 2003, pp 781-792

[7] M.F. Levin, On the nature and measurement of spasticity, in Clinical Neurophysiology, 2005. 116; 1754-1755

[8] J L. Bernhard, Towards a Clinical measurement of Spasticity. In Physiotherapy. 199581.

[9] J.W. Lance, Spasticity: Disorder of Motor Control. Chicago, Year Book Medical, 1980, pp. 485-500.

[10] P. Decq, P. Pilipetti, J.P. Lefaucheur, Evaluation of Spasticity in Adults. Operative Technique in Neurosurgery. 2005, PP.100-108.

[11] Vodovnik L, Bowman BR, Bajd T. Dynamics of spastic knee joint. Med Biol Eng Comput.1984;22(1):63-9.

[12] A.Jobin, M.F. Levin. Regulation of stretch reflex threshold in elbow flexors in children with Cerebral Palsy a new measure of spasticity. Developmental Medicine \& child Norology, 2000 42: pp.531-540 